\title{
Loss-of-Function ROX1 Mutations Suppress the Fluconazole Susceptibility of upc2A $\Delta$ Mutation in Candida glabrata, Implicating Additional Positive Regulators of Ergosterol Biosynthesis
} \author{
P. David Rogers, ${ }^{d}$ (D)W. Scott Moye-Rowley, ${ }^{b}$ (D) Damian J. Krysan ${ }^{a, e}$ \\ aDepartment of Pediatrics, Carver College of Medicine, University of lowa, lowa City, lowa, USA \\ bDepartment of Molecular Physiology and Biophysics, Carver College of Medicine, University of lowa, lowa City, lowa, USA \\ Institute of Life Sciences, Swansea University Medical School, Swansea University, Swansea, Wales, United Kingdom \\ dDepartment of Pharmaceutical Sciences, St. Jude Children's Research Hospital, Memphis, Tennessee, USA \\ eDepartment of Microbiology and Immunology, Carver College of Medicine, University of lowa, lowa City, lowa, USA
}

Tomye L. Ollinger, ${ }^{a}$ Bao Vu, ${ }^{b}$ Daniel Murante, ${ }^{a}$ Josie E. Parker, ${ }^{c}$ Lucia Simonicova, ${ }^{b}$ Laura Doorley, ${ }^{d}$ Mark A. Stamnes, ${ }^{b}$ Steven L. Kelly,

ABSTRACT Two of the major classes of antifungal drugs in clinical use target ergosterol biosynthesis. Despite its importance, our understanding of the transcriptional regulation of ergosterol biosynthesis genes in pathogenic fungi is essentially limited to the role of hypoxia and sterol-stress-induced transcription factors such as Upc2 and Upc2A as well as homologs of sterol response element binding (SREB) factors. To identify additional regulators of ergosterol biosynthesis in Candida glabrata, an important human fungal pathogen with reduced susceptibility to ergosterol biosynthesis inhibitors relative to other Candida spp., we used a serial passaging strategy to isolate suppressors of the fluconazole hypersusceptibility of a upc $2 A \Delta$ deletion mutant. This led to the identification of loss-of-function mutations in two genes: ROX1, the homolog of a hypoxia gene transcriptional suppressor in Saccharomyces cerevisiae, and CST6, a transcription factor that is involved in the regulation of carbon dioxide response in C. glabrata. Here, we describe a detailed analysis of the genetic interaction of ROX1 and UPC2A. In the presence of fluconazole, loss of Rox 1 function restores ERG11 expression to the upc2AD mutant and inhibits the expression of $E R G 3$ and $E R G 6$, leading to increased levels of ergosterol and decreased levels of the toxic sterol $14 \alpha$ methyl-ergosta-8,24(28)-dien-3 $\beta, 6 \alpha$ diol, relative to the upc2A $2 A$ mutant. Our observations establish that Rox 1 is a negative regulator of $E R G$ gene biosynthesis and indicate that a least one additional positive transcriptional regulator of $E R G$ gene biosynthesis must be present in $C$. glabrata.

IMPORTANCE Candida glabrata is one of the most important human fungal pathogens and has reduced susceptibility to azole-class inhibitors of ergosterol biosynthesis. Although ergosterol is the target of two of the three classes of antifungal drugs, relatively little is known about the regulation of this critical cellular pathway. Sterols are both essential components of the eukaryotic plasma membrane and potential toxins; therefore, sterol homeostasis is critical for cell function. Here, we identified two new negative regulators in C. glabrata of ergosterol (ERG) biosynthesis gene expression. Our results also indicate that in addition to Upc2A, the only known activator of $E R G$ genes, additional positive regulators of this pathway must exist.

Editor J. Andrew Alspaugh, Duke University Medical Center

Copyright $\odot 2021$ Ollinger et al. This is an open-access article distributed under the terms of the Creative Commons Attribution 4.0 International license.

Address correspondence to Damian J. Krysan, damian-krysan@uiowa.edu.

The authors declare no conflict of interest.

Received 5 October 2021

Accepted 1 December 2021

Published 22 December 2021 
andida species are a very common cause of human fungal infections. Candidiasis encompasses infections of mucosal and epidermal tissues such as oropharyngeal candidiasis and vulvovaginal candidiasis as well as invasive infections of deep organs and the bloodstream (1). Mucosal infections affect both immunocompetent and immunocompromised people while invasive infections primarily affect critically ill patients or those with altered immune function. Although Candida albicans is the most prevalent infecting species, the so-called non-albicans Candida spp., such as C. glabrata, C. parapsilosis (2) and, most recently, C. auris, have emerged as clinically significant causes of candidiasis (3). Of these, C. glabrata is the second most common cause of candidiasis in most clinical case series of invasive infections $(1,2)$.

One of the most important characteristics that distinguishes C. glabrata from C. albicans is that $C$. glabrata is less susceptible to azole antifungal drugs and has the highest rate of resistance to echinocandins; thus, C. glabrata has reduced susceptibility to two of the three classes of drugs currently used to treat invasive fungal infections (4). The rate of azole and echinocandin resistance varies significantly by institution (5). For example, some institutions report almost no resistance while others have fluconazole resistance rates as high as $18 \%$. In contrast, the rates of $C$. albicans azole resistance remain uniformly quite low. Genomic sequences of azole-resistant $C$. albicans isolates indicate that mutations in multiple genes can lead to resistance, including gain-offunction mutations in transcription factors regulating drug efflux pump expression, mutations in the azole target ERG11, and gain-of-function mutations in UPC2, a transcription factor that regulates the expression of ergosterol biosynthesis genes during periods of sterol depletion (6). Curiously, mutations associated with azole resistance in C. glabrata clinical isolates are essentially limited to gain-of-function alleles in PDR1, the transcription factor that regulates efflux pump expression, suggesting that these two species adapt to azole-induced sterol depletion in very different ways $(7,8)$.

Although gain-of-function mutations in the C. glabrata ortholog of C. albicans Upc2, $U P C 2 A$, have not been isolated in fluconazole-resistant C. glabrata clinical isolates, Whaley et al. showed that UPC2A was essential for fluconazole resistance in PDR1 gainof-function mutants (9). In the absence of sterol stress such as hypoxia or azole exposure, UPC2A has a modest effect on ergosterol gene (ERG) expression (9-11); however, under these inducers of sterol stress, upc $2 A \Delta$ mutants show a dramatic reduction in fitness $(9,10)$. Previous studies in C. glabrata indicate that Upc2A regulates genes involved in the uptake of exogenous sterols during hypoxic growth (11). However, little is known about how UPC2A interacts with other genes involved in ergosterol biosynthesis or the cellular response to sterol depletion. In Saccharomyces cerevisiae, the Upc2A homolog ScUpc2 functions with $S c E c m 22$ to regulate sterol biosynthesis (12). The upc $2 \Delta$ ecm $22 \Delta$ double mutant is viable but hypersusceptible to inhibitors of the ergosterol pathway and resistant to amphotericin B (12). In C. glabrata, Upc2B is homologous to Upc2A but upc2B $\Delta$ mutants do not show increased susceptibility to fluconazole or decreased growth under hypoxic conditions (10). These observations suggest that the transcriptional regulation of the ergosterol pathway in C. glabrata is distinct from that in the model yeast $S$. cerevisiae.

More generally, the regulation of ERG gene expression in fungal pathogens is remarkably understudied given the importance of the ergosterol biosynthesis pathway to the treatment of fungal infections (13); two of the three main therapies, azoles and polyenes, target ergosterol. To date, the main focus of this area of research has been on factors related to mammalian sterol response element binding (SREB) proteins or the Zinc(2)-Cys(6) factors such as Upc2A that function similarly to SREB factors but have no sequence homology to those proteins (14). Furthermore, if we consider the fact that these factors are not essential and have little effect on ergosterol levels or growth in the absence of sterol stress, then additional pathways and factors must regulate the expression of ERG genes.

To identify additional regulators of the ERG pathway under sterol stress, we took advantage of the fact that deletion of UPC2A prevents increased efflux pump 
expression from causing azole resistance and designed a laboratory evolution experiment for the isolation of suppressors of upc $2 A \Delta$ fluconazole hypersusceptibility (9). In wild-type cells, an experimental laboratory evolution approach in the presence of fluconazole would be expected to lead to gain-of-function mutants in Pdr1, the transcription factor (TF) that regulates the expression of efflux pumps CDR1/2. We hypothesized that the presence of a upc $2 A \Delta$ mutation would prevent the emergence of PDR1 gainof-function mutations and allow us to identify either gain-of-function mutants in other positive regulators of ERG gene expression or, alternatively, loss-of-function mutants in negative regulators.

As we describe below, this laboratory evolution strategy led to the isolation of lossof-function mutations in two transcription regulators: ROX1 and CST6. ROX1 is homologous to a repressor of hypoxia genes in S. cerevisiae (15), while CST6 is a homolog of an S. cerevisiae and a C. albicans (Rca1) transcriptional regulator of carbonic anhydrase expression (16). In this report, we focused our characterization on the transcriptional and biochemical changes that mediate the suppression of upc $2 A \Delta$ fluconazole hypersusceptibility by $R O X 1$ loss-of-function mutations; a detailed analysis of the interaction of CST6 with UPC2A awaits additional work.

\section{RESULTS}

Experimental laboratory evolution generates suppressors of upc $2 A \Delta$ fluconazole hypersusceptibility. The general strategy for our experimental laboratory evolution experiment is outlined in Fig. 1A. First, we constructed a upc2A $\Delta$ mutant in the His-BG2 genetic background (17) using a recyclable dominant selectable marker system recently reported by members of our team (18). Three founder colonies were selected, and 88 lineages from each were inoculated into a microtiter plate containing yeastpeptone-dextrose (YPD) medium supplemented with fluconazole at 1/8 MIC $(0.25 \mu \mathrm{g} /$ $\mathrm{ml}$ ) of the parental strains (Fig. 1). The cultures were grown to stationary phase and then passaged to a 2-fold-higher concentration of fluconazole until a final concentration of $64 \mu \mathrm{g} / \mathrm{ml}(32 \times \mathrm{MIC})$ was reached. All but 20 lineages went extinct before reaching a fluconazole concentration of $64 \mu \mathrm{g} / \mathrm{ml}$. The contents of wells that grew at $64 \mu \mathrm{g} /$ $\mathrm{ml}$ were plated on YPD as well as on synthetic medium lacking histidine to eliminate possible environmental contaminants. Inspection of the YPD plates indicated that the majority of wells contained heterogenous mixtures of small colonies and rarer large colonies, although some wells had large colonies only (Fig. 1B).

We purified both types of colonies by serially passaging on YPD plates without fluconazole three times. The small-colony isolates retained this colony morphology in the absence of selective pressure. These small colonies also grew poorly on glucose relative to the parental upc $2 A \Delta$ strains but were much more fit than the parental upc $2 A \Delta$ strains in the presence of fluconazole (Fig. 1C). The small colony size and poor growth on glucose strongly suggested that these strains might have mitochondrial defects (19). Consistent with that hypothesis, the small-colony isolates were unable to grow on glycerol plates and showed decreased mitochondrial DNA staining (data not shown), consistent with petite isolates. Previous work has shown that petite C. glabrata strains as well as those with other mitochondrial defects have increased Pdr1 activity, elevated expression of the Cdr1 efflux pump, and reduced fluconazole susceptibility (19).

From the large-colony isolates, 11 retained decreased fluconazole susceptibility relative to the parental upc2A $\Delta$ strains after serial passaging on YPD plates (Fig. 1D). The growth curves for two representative resistant isolates are provided in Fig. S1 in the supplemental material, further confirming the resistance of the strains to fluconazole relative to the upc $2 A \Delta$ strain. As discussed above, the most common mechanism of fluconazole resistance is Pdr1 gain-of-function mutations that lead to increased expression of Cdr1, a putative fluconazole efflux pump $(7,8)$. To determine if any of the 11 large-colony isolates had increased Cdr1 expression, we analyzed the protein levels of Cdr1 protein in the suppressor strains by Western blotting (Fig. 1E). Of the 11 suppressors, only two strains showed evidence of increased Cdr1 expression relative to the parental strain: the petite strain and $\mathrm{P} 1 \mathrm{~A} 1 \mathrm{~b}$, a nonpetite mutant. Thus, the decreased 
A

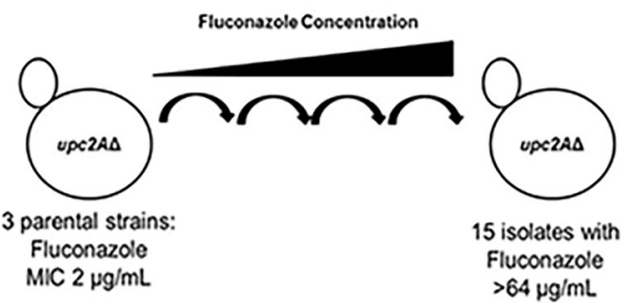

B

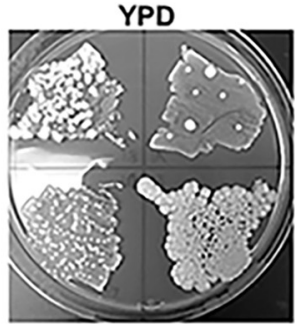

YPD

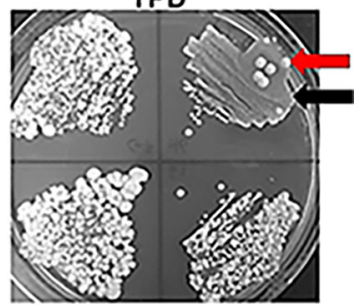

C

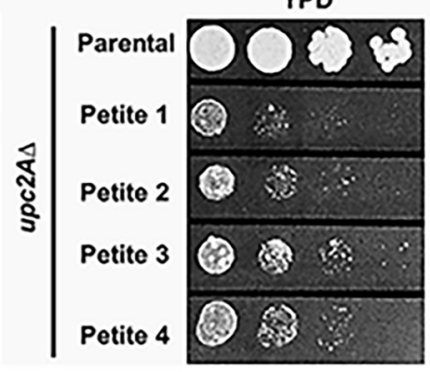

YPD + Fluconazole

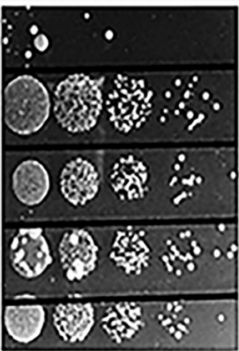

YPD

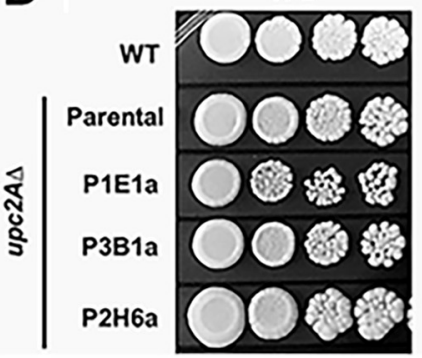

YPD + Fluconazole

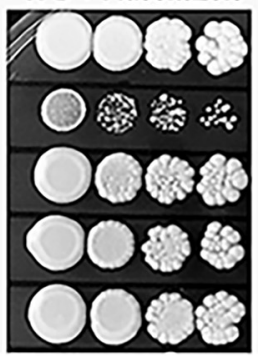

E

\section{$\alpha-C d r 1$}

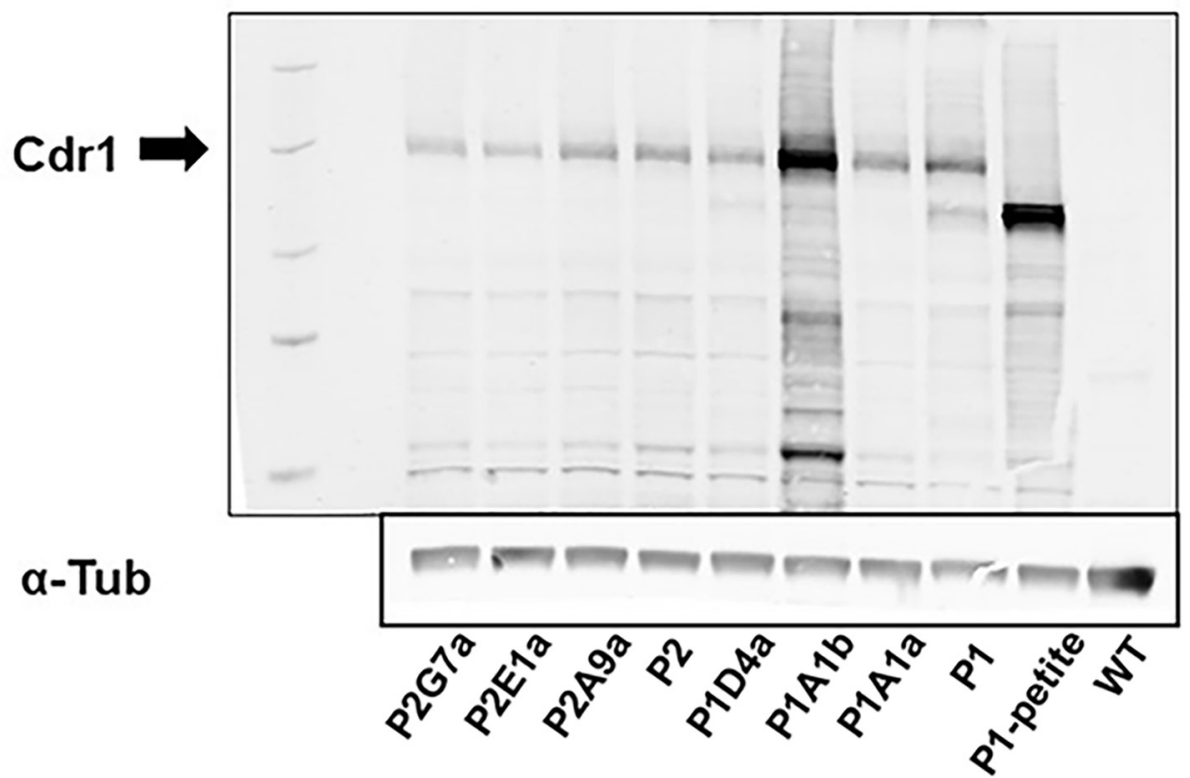

FIG 1 Experimental laboratory evolution identifies suppressors of upc2A $\Delta$ fluconazole hypersusceptibility. (A) Schematic of selection strategy. The parental strains were independent isolates of a upc2A $\Delta$ mutant generated in the BG2 background. The evolved isolates were coded as follows: P, parent 1, 2, or 3; column of well $A$ to $H$; row 1 to 12; and a or b isolate, e.g., P1E1a. (B) Representative plates inoculated with the contents of wells with growth at $64 \mu \mathrm{g} / \mathrm{ml}$ of fluconazole. The red arrow indicates a representative largecolony isolate, and the black arrow indicates a representative petite-colony isolate. (C) Spot dilution assays of representative petitecolony isolates on YPD and YPD $+40 \mu \mathrm{g} / \mathrm{ml}$ fluconazole. (D) Spot dilution assays of representative large-colony isolates on YPD and YPD $+40 \mu \mathrm{g} / \mathrm{ml}$ fluconazole. (E) Western blot analysis of representative large-colony and petite isolates blotted using a polyclonal anti-Cdr1 antibody after growth in YPD to log phase. The blot is representative of two biological replicates which showed the same pattern. The parental strains noted as P1 and P2 are upc2A $\Delta$ derivatives of BG2, which is noted as wild type in the blot. The P1-petite is a representative petite strain isolated from the P1 parent. An antitubulin antibody blot was used as a loading control.

fluconazole susceptibility for the majority (10/11) of the suppressor mutants cannot be explained by Pdr1-mediated efflux pump activation. As noted above, petite strains of C. glabrata activate Pdr1, and these data further support the assignment of these strains as petite (19). Previous experiments in a clinical C. glabrata isolate indicated that upc $2 A \Delta$ strongly reduced the effect of Pdr1 gain-of-function mutations on 
A

\begin{tabular}{|c|c|c|}
\hline Strain & Gene & Mutation \\
\hline P1A1b & CST6 & FS, S229 \\
\hline P1D4a & CST6 & FS, E34 \\
\hline P1D4a & PWP3 & G185P \\
\hline P1E1a & CST6 & NS, T200 \\
\hline P2A9a & CST6 & NS, Q148 \\
\hline P2E1a & ROX1 & FS, P41 \\
\hline P2G7a & CST6 & FS, N72 \\
\hline
\end{tabular}

B Mutations

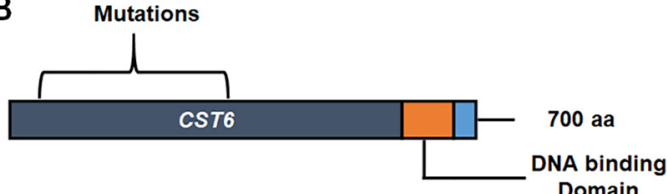

\begin{tabular}{|c|c|c|}
\hline Strain & Gene & Mutation \\
\hline P2H6a & ROX1 & $\begin{array}{c}\text { Stop, } \\
\text { W217 }\end{array}$ \\
\hline P2H6a & PWP3 & Q266L \\
\hline P2A1a & ROX1 & NS, E216 \\
\hline P3B1a & ROX1 & NS, S60 \\
\hline P3B4a & CST6 & NS, Q121 \\
\hline P3B4a & HXT4 & M845V \\
\hline P3D9a & MED6 & NS, T3 \\
\hline
\end{tabular}

Domain

C

Mutations

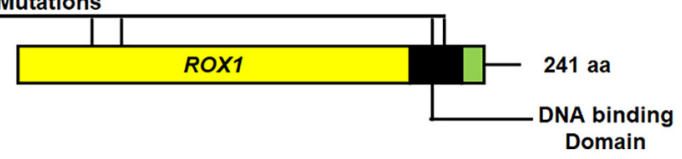

FIG 2 Loss-of-function mutations in ROX1 and CST6 are present in the upc2AD fluconazolesuppressor strains. (A) The table indicates the code for the individual isolate and the coding region mutation identified in that isolate. Isolates containing CST6 mutations are indicated by blue-shaded rows, and those containing ROX1 mutations are indicated by boldface on a gray background. Additional mutations are in lightface on gray backgrounds without boldface. (B) Location of mutations in CST6. (C) Location of mutations in ROX1. aa, amino acids.

fluconazole susceptibility (9). Our observations, however, indicate that under some circumstances Pdr1 activation can lead to Upc2A-independent reduction in fluconazole susceptibility but that upc2A $A$ mutation largely prevents the emergence of $P D R 1$ gainof-function mutations as a mechanism of fluconazole resistance.

Genomic sequencing identifies putative loss-of-function mutations in ROX1 and

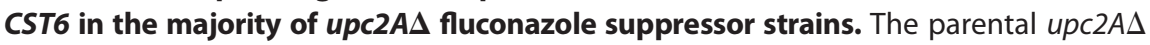
founder strains as well as 12 suppressor strains were resequenced. Following analysis using the Lasergene suite of software, 11 strains were found to contain mutations within coding regions (Fig. 2A). Of these 11, 6 contained either frameshift or nonsense mutations that would lead to loss of function in CAGL0I05170g (17, 20), a homolog of S. cerevisiae transcription factor SCCST6 (C. glabrata gene and protein names do not have prefix modifiers here while $C$. albicans and $S$. cerevisiae genes and protein names do have species prefixes). Four strains contained similar types of loss-of-function mutations in CAGLOD05434g, homologous to the S. cerevisiae high-mobility group (HMG) transcriptional repressor $\operatorname{ROX} 1(15,17)$. One strain contained a nonsense codon in CAGLOD03828g, an uncharacterized homolog of the S. cerevisiae mediator component, MED6; this is predicted to be an essential protein and was not pursued further. One rox 1 and one cst6 mutant strain also had additional nonsynonymous polymorphism in the cell surface adhesion gene PWP3. Finally, a cst6 mutant strain also had a nonsynonymous polymorphism in the putative glucose transporter HXT4.

In S. cerevisiae, ScCst6 has been shown to regulate the expression of carbonic anhydrase (NCE103), and deletion mutants of CST6 have similarly been shown to play a modest role in the expression of $N C E 103$ and $\mathrm{CO}_{2}$ sensing in C. glabrata (21). In C. albicans, CaRCA1 is the homolog of SCCST6 and CST6. CaRCA1 plays a role in NCE102 expression (21) but also appears to affect ergosterol biosynthesis as evidenced by the observations that Carca $1 \Delta \Delta$ mutants have decreased susceptibility to fluconazole and reduced levels of ergosterol relative to the wild type (WT) (22). A library of $C$. glabrata deletion mutants contains a cst6 $\Delta$ strain, but it was not reported to have altered susceptibility to fluconazole. The putative CST6 loss-of-function mutations identified in 

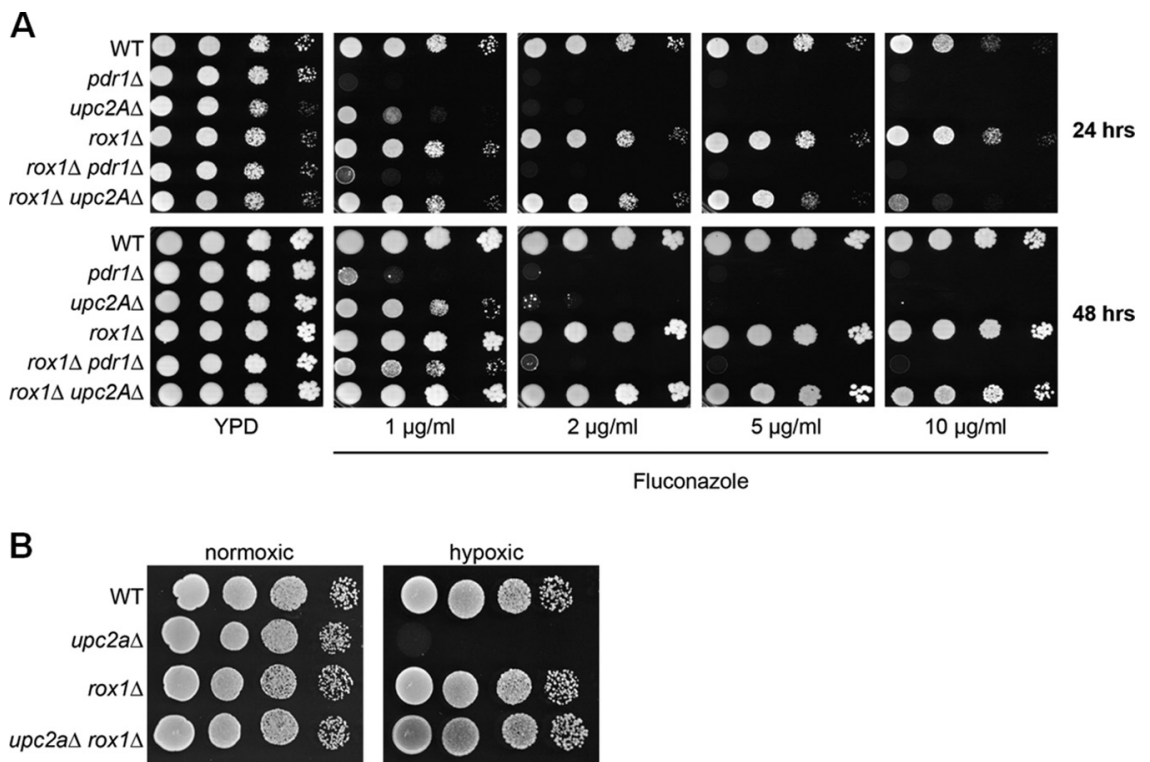

FIG 3 Deletion of $R O X 1$ suppresses fluconazole and hypoxia hypersusceptibility of upc $2 A \Delta$ mutants. (A) Tenfold dilution series of the indicated strains were spotted on YPD or YPD plates with the indicated concentrations of fluconazole and incubated for 24 or $48 \mathrm{~h}$ at $30^{\circ} \mathrm{C}$. (B) The indicated strains were plated on YPD and incubated at $30^{\circ} \mathrm{C}$ in ambient air or in a GasPak to establish a lowoxygen environment as described in Materials and Methods.

these strains are frameshift or nonsense mutations within the N-terminal third of the protein, well short of the basic leucine zipper (bZip) DNA binding domain (Fig. 2B).

In S. cerevisiae, Rox1 is a well-studied HMG repressor of hypoxia-related genes including ergosterol biosynthesis genes. Indeed, ScRox 1 functions to repress some genes that are activated by ScUpc2 and its ortholog ScEcm33 in response to either ergosterol depletion or reduced oxygen availability (23). For example, previous work from the Edlind lab has shown that deletion of SCROX1 decreases the susceptibility of $S$. cerevisiae to azole antifungals 4- to 10-fold and increases the expression of ERG genes (24). However, the role of $R O X 1$ in C. glabrata has not been investigated previously besides as part of the large-scale library screening (17). This screen found that the rox $1 \Delta$ mutant was more susceptible to amphotericin but similar to wild type in the presence of fluconazole. Interestingly, although both ScRox1 and Rox1 contain homologous DNA binding domains, the HMG DNA binding domain for SCRox 1 is positioned at the $\mathrm{N}$ terminus, while the DNA binding domain of Rox 1 is at the $C$ terminus (Fig. $2 \mathrm{C}$ ). The ROX1 mutations are nonsense mutations either early in the $\mathrm{N}$-terminal portion or within the DNA binding domain (Fig. 2C).

Loss-of-function mutations of ROX1 suppress fluconazole hypersusceptibility of upc $2 A \Delta$ strains. To confirm that the loss-of-function mutations identified in the upc $2 A \Delta$ fluconazole suppressor strains were responsible for this phenotype, we attempted to make the corresponding double mutant deletion strains. In the case of $R O X 1$, we were able to generate the rox $1 \Delta$ and $\operatorname{rox} 1 \Delta$ upc2A strains in the KKY2001 background; in addition, we generated a rox $1 \Delta$ mutation in a strain lacking PDR1, a transcription factor that has a profound effect on fluconazole susceptibility. In the presence of fluconazole, upc $2 A \Delta$ and pdr $1 \Delta$ cells have a significant growth defect relative to WT while the rox $1 \Delta$ strain is slightly less susceptible at high concentrations of fluconazole after $24 \mathrm{~h}$ but not at $48 \mathrm{~h}$ (Fig. 3A). Deletion of ROX1 in the pdr1 $\mathrm{B}$ background nearly restores WT growth at the lowest concentrations of fluconazole after $48 \mathrm{~h}$ but has minimal effect on the increased fluconazole susceptibility of the $p d r 1 \Delta$ mutant at higher concentrations. In contrast, deletion of $R O X 1$ in the upc $2 A \Delta$ mutant restores growth to a near-wild-type rate at the lower concentrations of fluconazole and at longer incubation times. This strongly supports the conclusion that the loss-of-function mutations in ROX1 identified in the evolved strains are responsible for the reduced fluconazole susceptibility relative to the upc $2 A \Delta$ 
parental strains. In addition, these data indicate that loss of Rox 1 function is not a global suppressor of fluconazole susceptibility since the mutation has minimal effects on WT or pdr1 $1 \Delta$ cells.

Despite multiple attempts to generate a $\operatorname{cst} 6 \Delta u p c 2 A \Delta$ double mutant using both standard recombination and CRISPR/Cas9 approaches, we were unable to do so. The cst6 $\Delta$ deletion mutant was generated as part of the large library of C. glabrata deletion mutants. In addition, others have reported the construction of cst6 $\Delta$ mutants in other contexts (20). We obtained the cst6s strain from the library collection and determined its susceptibility to fluconazole; in CLSI microdilution assays, it is less susceptible to fluconazole than the parental strain (MIC for cst6 $\Delta$ strain, $16 \mu \mathrm{g} / \mathrm{ml}$, versus WT, $4 \mu \mathrm{g} / \mathrm{ml}$ ). We elected to focus the rest of our characterization on the interaction between Rox 1 and Upc $2 A$ since we were able to confirm that loss of function of $R O X 1$ suppresses the upc $2 A \Delta$ fluconazole hypersusceptibility phenotype.

Upc2A is also required for C. glabrata to grow under hypoxic conditions $(10,11)$, and ScRox 1 was originally identified as a repressor of hypoxia-related genes during growth under aerobic conditions (23). Accordingly, many genes repressed by ScRox1 during normoxia are also activated by $S c U p c 2$. Therefore, we compared the growth of the upc $2 A \Delta \operatorname{rox} 1 \Delta$ strain to that of upc $2 A \Delta$ and $\operatorname{rox} 1 \Delta$ strains under low-oxygen conditions (Fig. 3B). As expected, the upc2A $\Delta$ mutant grew poorly while the rox $1 \Delta$ mutant grew similar to the wild type. Consistent with its fluconazole phenotype, the $\operatorname{rox} 1 \Delta$ upc $2 A \Delta$ mutant grew similarly to the wild type, indicating that $R O X 1$ loss of function suppressed both the fluconazole and hypoxia susceptibility of the upc $2 A \Delta$ strain.

Deletion of ROX1 increases ergosterol content and alters the sterol profile of upc $2 \boldsymbol{A} \Delta$ mutants. To gain insights into how rox $1 \Delta$ affects the sterol content and distribution (Fig. 4A) in the presence and absence of fluconazole, we used mass spectrometry to characterize the sterol profile of WT, upc $2 A \Delta, \operatorname{rox} 1 \Delta$, and $\operatorname{rox} 1 \Delta$ upc $2 A \Delta$ strains in YPD medium with and without $8 \mu \mathrm{g} / \mathrm{ml}$ fluconazole. In the absence of fluconazole, WT, $\operatorname{rox} 1 \Delta$, and upc2A $\Delta$ strains had statistically indistinguishable amounts of total cell ergosterol while the rox $1 \Delta$ upc2A $\Delta$ strain had $\sim 1.5$-fold more ergosterol than the other strains (Fig. 4B). In the presence of fluconazole concentrations that allowed the upc $2 A \Delta$ strian to grow, albeit at a reduced rate (Fig. S1), the rox $1 \Delta$ strain had modestly increased ergosterol while the ergosterol content of the upc $2 A \Delta$ mutant was reduced slightly (Fig. 4C). The rox $1 \Delta$ upc2A $\Delta$ double mutant, in contrast, had nearly $50 \%$ more ergosterol than the upc $2 A \Delta$ deletion mutant and had statistically significantly increased ergosterol content over that of WT.

We also determined the relative ratios of the different intermediate and alternative pathway sterols for the mutants with and without fluconazole exposure. The ergosterol biosynthesis intermediate (Fig. $4 \mathrm{~A}$ ) that is most altered in the upc $2 A \Delta \operatorname{rox} 1 \Delta$ mutant in both the presence (Fig. 4D) and absence of fluconazole is zymosterol (Table S1), a substrate for the $\mathrm{C}_{24}$ methyltransferase enzyme Erg6. Zymosterol accumulates in both $\operatorname{rox} 1 \Delta$ and upc $2 A \Delta \operatorname{rox} 1 \Delta$ strains in the absence of fluconazole (Table S1) but does so in the double mutant only in the presence of fluconazole (Fig. 4D). These data suggest that Erg6 activity is reduced in strains lacking ROX1 and particularly in fluconazoletreated rox $1 \Delta$ upc2As mutants. Erg6 also catalyzes the conversion of lanosterol, the substrate of Erg11, into eburicol, the first sterol in the alternate pathway (Fig. 4A).

Lanosterol is a minor component of total sterols in the absence of fluconazole (WT/ YPD: $1.28 \%$, Table S1) but increases dramatically in fluconazole-treated cells (WT/FLC: $20 \%$, Table S1) because the activity of Erg11 is inhibited. As shown in Fig. 4E, lanosterol is further increased in fluconazole-treated $\operatorname{rox} 1 \Delta$ upc $2 A \Delta$ mutants relative to fluconazole-treated WT. Since ergosterol is not depleted in the $\operatorname{rox} 1 \Delta$ upc $2 A \Delta$ mutant, the increase in lanosterol seems unlikely to be due to differences in Erg11 activity but rather to reduced Erg6-mediated conversion of lanosterol to eburicol (Fig. 4A). Further supporting this notion, eburicol is significantly reduced in fluconazole-treated $\operatorname{rox} 1 \Delta$ upc $2 A \Delta$ cells relative to WT (Fig. $4 \mathrm{~F}$ ). These data strongly support the idea that the combination of upc $2 A \Delta$ and $\operatorname{rox} 1 \Delta$ mutations leads to reduced Erg6 activity in the presence of fluconazole. 
A

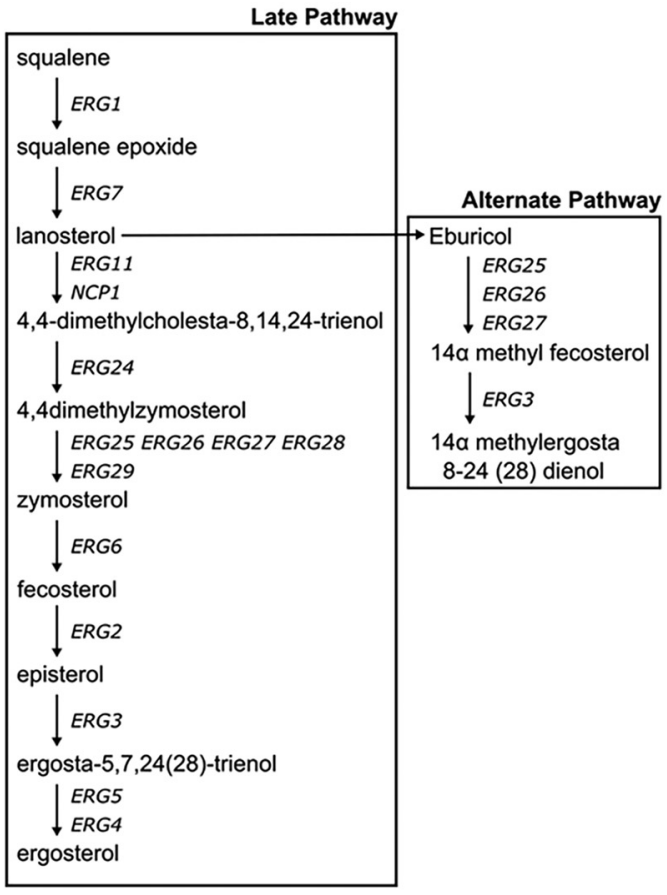

B

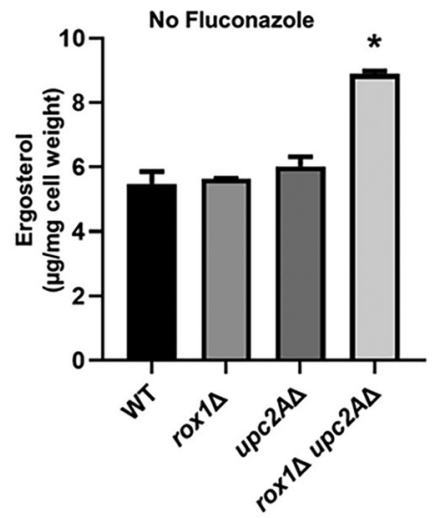

C

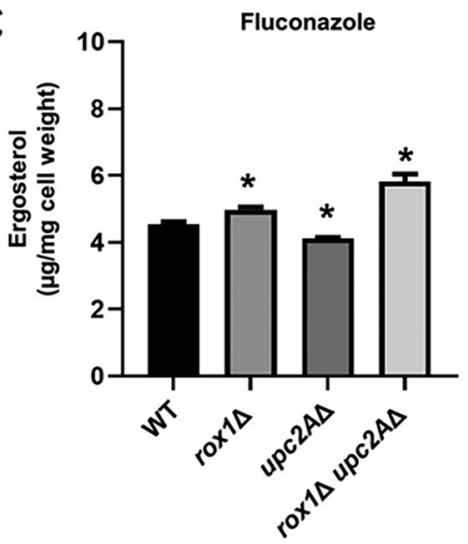

G

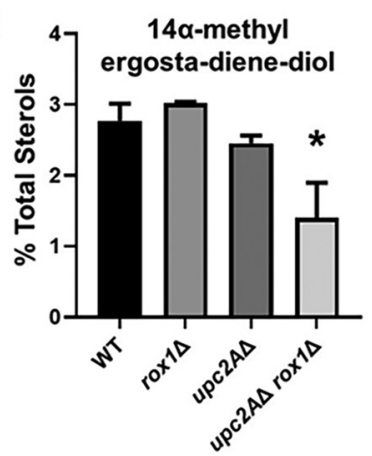

E

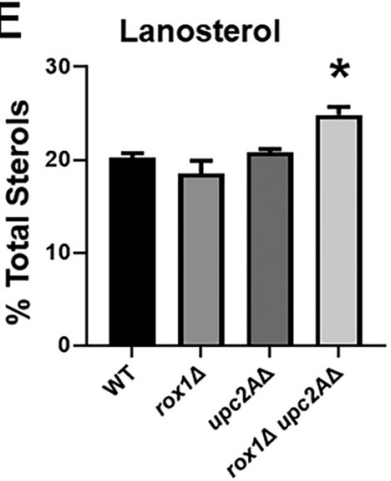

H

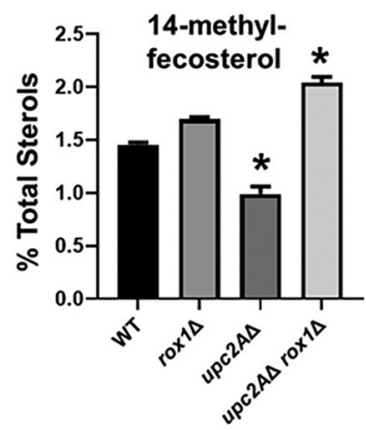

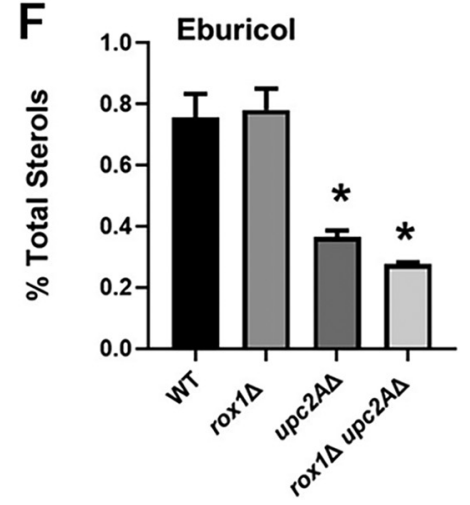

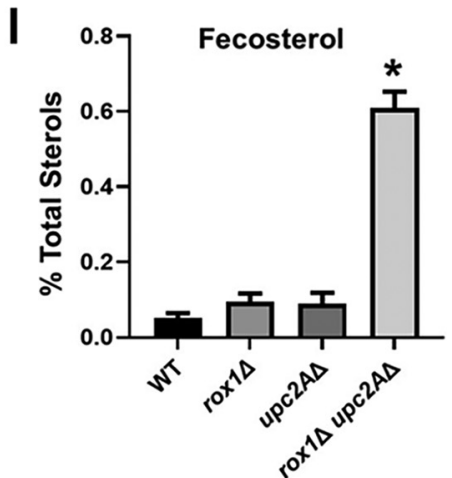

FIG 4 The rox1 $1 \Delta$ upc2As mutants have altered sterol profiles. (A) Schematic of the late portion of the ergosterol biosynthesis pathway. (B and C) The content of ergosterol $(\mu \mathrm{g} / \mathrm{mg}$ cell weight) was determined as described in Materials and Methods from log-phase YPD cultures of the indicated strains without (B) and with (C) fluconazole $(8 \mu \mathrm{g} / \mathrm{ml})$. (D to I) The percentage of the indicated sterols relative to total sterols for the indicated strains was determined for fluconazole-treated 
Ultimately, the alternate pathway converts lanosterol to $14 \alpha$ methyl-ergosta-8,24 (28)-dien-3 $\beta, 6 \alpha$-diol (Fig. 4A), which is toxic to cells and is thought to be part of the mechanism by which azole drugs inhibit fungal growth (25). In rox $1 \Delta$ upc $2 A \Delta$ mutants treated with fluconazole, the toxic dienol is reduced relative to upc $2 A \Delta$ and WT strains (Fig. 4G). In addition, the proportion of $14 \alpha$ methyl fecosterol, the immediate precursor to $14 \alpha$ methyl-ergosta-8,24(28)-dien-3 $\beta, 6 \alpha$-diol, is also increased in $\operatorname{rox} 1 \Delta$ upc $2 A \Delta$ mutants treated with fluconazole (Fig. 4H). Erg3 catalyzes this conversion (Fig. 4A), and the accumulation of $14 \alpha$ methyl fecosterol in the fluconazole-treated upc $2 A \Delta$ rox $1 \Delta$ double mutant suggests that Erg3 activity may also be reduced in that strain.

In the ergosterol pathway, Erg3 also converts episterol to ergosta-5,7,24(28)-trienol. Although episterol is elevated in the fluconazole-treated $\operatorname{rox} 1 \Delta$ upc $2 A \Delta$ mutant, the variation between samples is too great to confidently conclude that it accumulates (Table S1). The previous sterol in the pathway is fecosterol, and its levels are increased 5 -fold in the fluconazole-treated $\operatorname{rox} 1 \Delta$ upc $2 A \Delta$ mutant relative to fluconazole-treated wild type (Fig. 4l). Because Erg3 mediates the conversion of fecosterol to episterol, it is likely that its reduced activity leads to the accumulation of fecosterol as well. Taken together, these data indicate that the $\operatorname{rox} 1 \Delta$ upc $2 A \Delta$ double mutant appears to have both increased overall ergosterol and reduced accumulation of the toxic diol relative to the upc $2 A \Delta$ strain in the presence of fluconazole. The sterol profiles suggest that the near-WT susceptibility of the upc $2 A \Delta \operatorname{rox} 1 \Delta$ strain to fluconazole may be due to alterations in the activity of Erg6 and Erg3. Since both Rox1 and Upc2A are transcriptional regulators of $E R G$ genes, it seems likely that the mechanism of this remodeling of the $\operatorname{rox} 1 \Delta$ upc $2 A \Delta$ strain's sterol composition is due, at least in part, to differential expression of genes such as ERG3 and ERG6.

ERG11 gene expression is maintained at WT levels in the rox $1 \Delta$ upc $2 A \Delta$ strain while ERG3 and ERG6 expression is reduced in the presence of fluconazole. We hypothesized that altered expression of ERG genes such as ERG3, ERG6, and ERG11 might be responsible for the changes in sterol content in $\operatorname{rox} 1 \Delta$ upc $2 A \Delta$ mutants exposed to fluconazole. To test that hypothesis, we determined the expression of ERG1-11\&24 in WT, upc2As, rox $1 \Delta$, and $\operatorname{rox} 1 \Delta$ upc $2 A \Delta$ strains in the presence and absence of fluconazole using quantitative reverse transcription-PCR (qRT-PCR) (Fig. 5A). A notable feature of the effect of these mutations on ERG gene expression is that it varies with the specific ERG gene. In the presence of fluconazole, the upc2A $\Delta$ mutant has the most consistent reduction in ERG gene expression with all but ERG5 and ERG7 having reduced expression relative to fluconazole-treated WT cells. This is as expected from previously reported data.

In the absence of fluconazole, ERG11 expression is elevated 3.5-fold in the rox $1 \Delta$ upc $2 A \Delta$ mutant relative to WT (Fig. $5 A$ and B). Since this strain lacks Upc2A, the only known regulator of $E R G$ gene expression in Candida, the elevated expression of ERG11 indicates that at least one other positive transcriptional regulator must exist. This increase in ERG11 expression correlates well with the increase in ergosterol content observed for this strain when grown in YPD (Fig. 4B). In the presence of fluconazole (Fig. 5A and B), ERG11 expression in the $\operatorname{rox} 1 \Delta$ upc2A $A$ strain is nearly identical to WT but reduced somewhat relative to the untreated strain, indicating that loss of Upc $2 \mathrm{~A}$ function has an effect on the expression of ERG11. Western blot analysis (Fig. 5C) confirmed the RNA levels as shown in Fig. 5B. Thus, the double mutant restores the expression of the fluconazole target Erg11 in the upc2As background to a level similar to that of the wild type, providing at least a partial explanation for the ability of rox $1 \Delta$ deletion to suppress the upc2A $\Delta$ fluconazole hypersusceptibility.

FIG 4 Legend (Continued)

samples. (D) Zymosterol. (E) Lanosterol. (F) Eburicol. (G) $14 \alpha$-Methyl-ergosta-diene-diol is a shortened name for $14 \alpha$ methylergosta-8,24(28)-dien-3 $\beta, 6 \alpha$-diol. (H) 14-Methyl fecosterol. (I) Fecosterol. The bars indicate the means with standard errors of the means. Statistical significance relative to wild type is indicated by an asterisk. Data were analyzed by analysis of variance (ANOVA) followed by Student $t$ tests with Bonferroni adjustment for individual comparisons with $P<0.05$ indicating statistical significance. The complete sterol data are provided in Table S1. 
A

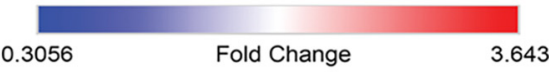

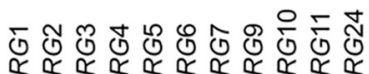

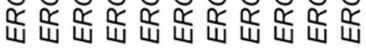

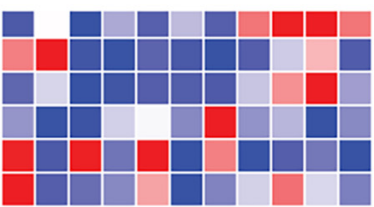

\begin{tabular}{l|l}
$\operatorname{upc} 2 A \Delta$ & - Flu \\
$\operatorname{rox} 1 \Delta$ & \\
$u p c 2 A \Delta \operatorname{rox} 1 \Delta$ & \\
$u p c 2 A \Delta$ & \\
$\operatorname{rox} 1 \Delta$ & + Flu \\
$u p c 2 A \Delta \operatorname{rox} 1 \Delta$ &
\end{tabular}

C
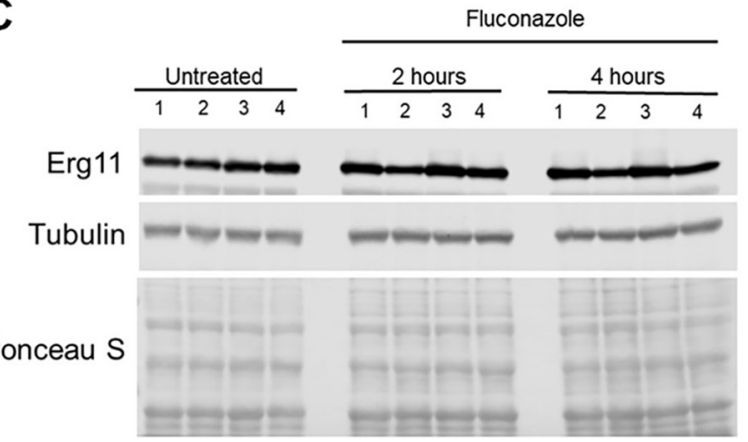

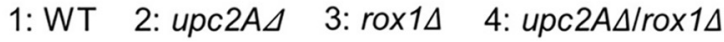

B

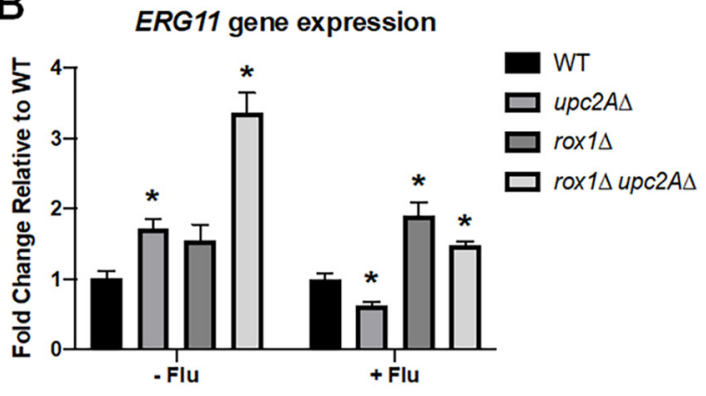

D

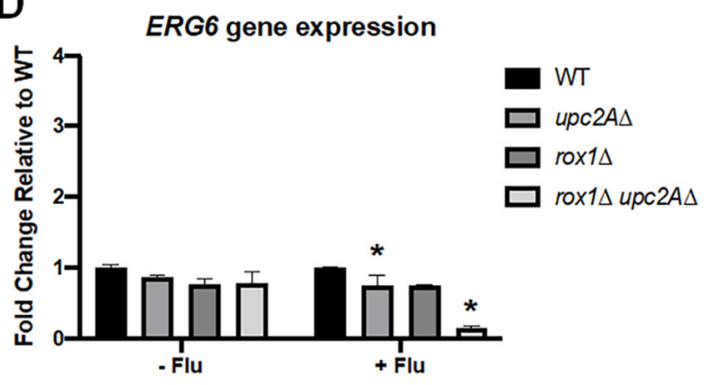

$\mathbf{E}$

ERG3 gene expression

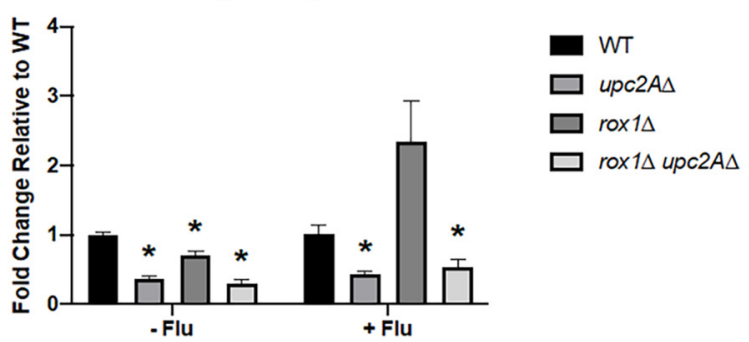

FIG 5 Deletion of rox $1 \Delta$ in the upc2AD mutant restores ERG11 expression to nearly wild-type levels in the presence of fluconazole.

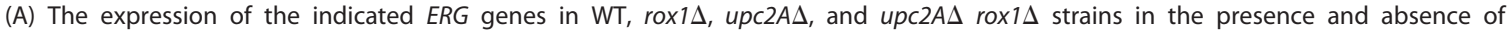
fluconazole $(8 \mu \mathrm{g} / \mathrm{ml})$ was determined by quantitative RT-PCR and is normalized to WT and summarized in a heat map. (B) The expression of ERG11 in the indicated strains. (C) Western blot of the indicated strains grown in YPD or YPD $+8 \mu \mathrm{g} / \mathrm{ml}$ fluconazole for $4 \mathrm{~h}$. (D) Expression of ERG6 in the indicated strains. (E) Expression of ERG3 in the indicated strains. The strains were incubated in YPD or YPD $+8 \mu \mathrm{g} / \mathrm{ml}$ fluconazole for $4 \mathrm{~h}$. The bars are means from three biological replicates performed in technical triplicate. The error bars indicate standard deviation, and Student's $t$ test was used to determine statistical significance. Statistical significance relative to wild type is indicated by an asterisk.

Overall, 8 of the 11 ERG genes that we examined are expressed in the rox $1 \Delta$ upc2AD mutant at levels that correspond to $\geq 75 \%$ of $W T$ in the presence of fluconazole (Fig. 5A). Of these 8, ERG11, ERG2, and ERG3 are reduced by at least 2-fold in the fluconazole-treated upc2A $\triangle$ mutant. Surprisingly, ERG6 is not significantly reduced in either

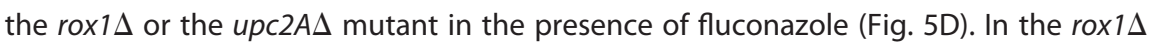
upc $2 A \Delta$ mutant, however, ERG6 expression is dramatically reduced, indicating that there is a strong negative genetic interaction between upc2A $\Delta$ and rox $1 \Delta$ mutations with respect to $E R G 6$ expression. As discussed above, the reduced expression of ERG6 in the fluconazole-treated $\operatorname{rox} 1 \Delta$ upc $2 A \Delta$ mutant is consistent with the sterol profiling data indicating decreased flux through that enzyme.

The expression of ERG3 is also reduced in the fluconazole-treated rox $1 \Delta$ upc2A $\Delta$ mutant (Fig. 5A and E) and correlates with sterol profiling evidence of reduced flux through that enzyme as well. ERG3 is upregulated in the rox $1 \Delta$ mutant in the presence 
of fluconazole and downregulated in the upc2A $\Delta$ mutant (Fig. 5E). In contrast to ERG11, however, the derepression of ERG3 in the rox $1 \Delta$ mutant does not compensate for its reduced expression in the upc $2 A \Delta$ mutant, and indeed, its expression remains reduced in the rox $1 \Delta$ upc2A mutant (Fig. 5E). This provides further support for the conclusion that the relationship between Rox 1 and Upc $2 A$ is more complex than a repressor and activator of a shared set of genes. However, the wild-type levels of ERG11 expression in the fluconazole-treated rox $1 \Delta$ upc $2 A \Delta$ mutant coupled with the reduced expression of $E R G 3$ and $E R G 6$ provide a potential mechanism for the ability of $\operatorname{rox} 1 \Delta$ to suppress upc $2 A \Delta$ fluconazole hypersusceptibility.

Transcriptional profiling of upc $2 A \Delta, \operatorname{rox} 1 \Delta$, and upc $2 A \Delta \operatorname{rox} 1 \Delta$ strains. To characterize the effect of the double mutant on genome-wide gene expression, we performed transcriptome sequencing (RNA-Seq) on upc $2 A \Delta$, rox $1 \Delta$, and upc $2 A \Delta \operatorname{rox} 1 \Delta$ strains in the presence and absence of fluconazole. The processed data sets for all strains and conditions are provided in Table S2. The trends for ERG gene expression observed in our single-gene analysis were consistent with those found in the genomewide expression profiling. The role of ROX1 in C. glabrata gene expression has not previously been characterized. As discussed above, its role in the repression of hypoxia-related genes during normoxia is well characterized in $S$. cerevisiae $(15,23)$. In log-phase growth in YPD, 90 genes are upregulated by 2 -fold with a corrected $P$ value of $\leq 0.05$ (Fig. 6A). As shown in Fig. 6B, 5 genes involved in hypoxic response were upregulated, suggesting that Rox 1 function is conserved between C. glabrata and S. cerevisiae. Previously reported transcriptional profiling indicated that ScRox 1 suppresses cell wall-related genes (23). Consistent with those findings, cell wall genes were enriched (GO term, corrected $P=3.4 \times 10^{-4}$; Fig. $6 \mathrm{C}$ ), further supporting conservation of function between ScRox 1 and Rox1.

In the presence of fluconazole, Rox 1 would be expected to no longer repress genes required for the cellular response to sterol depletion, and thus, those genes may not be differentially regulated in the deletion mutant relative to WT. In the presence of fluconazole, 93 genes are upregulated in the rox $1 \Delta$ mutant. Of the 93 genes, 48 remain upregulated in the presence of fluconazole while 42 are no longer upregulated; the latter set would be candidate genes that may be Rox 1 regulated in the presence of sterol stress. This set of genes is enriched for carbohydrate metabolic processes $\left(P=7.7 \times 10^{-4}\right)$ and the oxidation-reduction process $\left(P=3.1 \times 10^{-3}\right)$, two classes of genes also found to be Scrox 1 dependent during sterol stress (23). The genes upregulated only in the presence of fluconazole were not enriched for any process or cellular compartment by GO term analysis while those in common were enriched in cell wall-related genes $\left(P=9.25 \times 10^{-6}\right)$ and included TIR3, a cell wall gene implicated in sterol uptake $(11,26)$.

The effect of the upc $2 A \Delta$ mutation on gene expression in the presence and absence of fluconazole has recently been reported by $\mathrm{Vu}$ et al. (27), and our results are consistent with those data. We used our upc2A $A$ strain data for comparison with the rox $1 \Delta$ upc $2 A \Delta$ strain (Fig. $7 \mathrm{~A}$ ) in the presence of fluconazole to determine whether non-ERG genes that were downregulated in the upc $2 A \Delta$ strain were returned to WT levels of expression by the deletion of ROX1. Nineteen of the 32 genes downregulated in the fluconazole-treated upc $2 A \Delta$ mutant were either equal to WT expression or upregulated in the rox $1 \Delta$ upc $2 A \Delta$ mutant. As expected, this set of genes was enriched for ergosterol biosynthesis genes $\left(P=5.8 \times 10^{-9}\right)$. In addition, UMP biosynthesis genes (corrected $\left.P=8.8 \times 10^{-8}\right)$ and oxidation-reduction genes $\left(P=2.8 \times 10^{-5}\right)$ were enriched, although it is difficult to connect these genes to fluconazole response. Thus, it appears that loss of Rox 1 function restores the expression of some Upc2A-dependent genes to levels comparable to WT cells in the presence of fluconazole. Recently, the regulon for Upc2A has been characterized using chromatin immunoprecipitation sequencing (ChIP-seq) (27). Using these data, we asked what proportion of genes that are upregulated in the $\operatorname{rox} 1 \Delta$ upc $2 A \Delta$ mutant relative to the upc $2 A \Delta$ mutant are direct targets of Upc2A. As shown in Fig. 7B, 23 of the 115 genes upregulated by deletion of ROX1 in 
A rox1 $\triangle$ relative to $W T$ in YPD

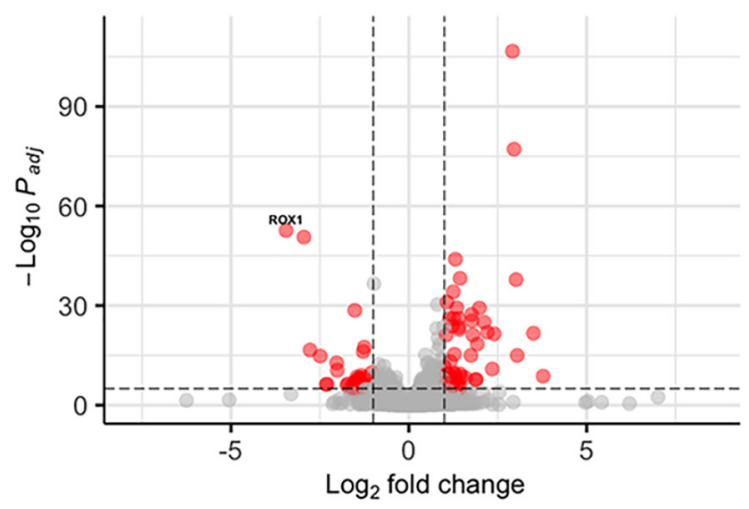

\section{B}

\begin{tabular}{|c|c|c|c|}
\hline ORF & $\begin{array}{c}\text { Gene } \\
\text { Name }\end{array}$ & Function & $\begin{array}{c}\text { rox14/WT } \\
\text { Fold } \\
\text { change }\end{array}$ \\
\hline CAGL0C03872g & TIR3 & $\begin{array}{c}\text { GPI, sterol } \\
\text { uptake }\end{array}$ & 9.1 \\
\hline CAGL0F01419g & AUS1 & $\begin{array}{c}\text { Sterol } \\
\text { transporter }\end{array}$ & 3.4 \\
\hline CAGL0K12100g & HEM13 & $\begin{array}{c}\text { Heme } \\
\text { Biosynthesis }\end{array}$ & 3.4 \\
\hline CAGL0K03003g & MOT3 & $\begin{array}{c}\text { Transcription } \\
\text { Factor, }\end{array}$ & 2.5 \\
\hline CAGL0B03421g & HAP1 & $\begin{array}{c}\text { Hypoxia } \\
\text { Transcription } \\
\text { Factor, } \\
\text { Hypoxia }\end{array}$ & 2.6 \\
\hline & & \begin{tabular}{l} 
Hypoxian \\
\hline
\end{tabular} & \\
\hline
\end{tabular}

C Cell wall genes upregulated in rox1 $1 \Delta$

\begin{tabular}{|c|c|c|c|}
\hline ORF & $\begin{array}{l}\text { Gene } \\
\text { Name }\end{array}$ & Function & $\begin{array}{c}\text { rox1 } 1 \Delta / W T \\
\text { Fold } \\
\text { change }\end{array}$ \\
\hline CAGL0B05093g & AWP9 & adhesin & 4.90 \\
\hline CAGLOC03872g & TIR3 & $\begin{array}{l}\text { GPI, sterol } \\
\text { uptake }\end{array}$ & 9.1 \\
\hline CAGL0F01485g & TIR2 & $\begin{array}{l}\text { GPI linked } \\
\text { cell wall }\end{array}$ & 2.2 \\
\hline CAGLOG10219g & AWP12 & adhesin & 2.3 \\
\hline CAGLOH08844g & none & $\begin{array}{l}\text { Putative } \\
\text { adhesin }\end{array}$ & 2.1 \\
\hline CAGLOH09592g & none & $\begin{array}{l}\text { GPI linked } \\
\text { cell wall }\end{array}$ & 2.2 \\
\hline CAGLOH09614g & none & $\begin{array}{l}\text { GPI linked } \\
\text { cell wall }\end{array}$ & 3.1 \\
\hline CAGLOH10626g & AWP13 & adhesin & 3.5 \\
\hline CAGL0J01463g & none & $\begin{array}{l}\text { GPI linked } \\
\text { cell wall }\end{array}$ & 3.4 \\
\hline CAGLOM00110g & none & adhesin & 3.0 \\
\hline
\end{tabular}

FIG 6 Rox1 represses the expression of hypoxia and cell wall-related genes under normoxia. (A) Volcano plot comparing the expression of genes in the rox $1 \Delta$ mutant to that in the WT in log-phase cultures incubated for $4 \mathrm{~h}$ in YPD at $30^{\circ} \mathrm{C}$. Red indicates genes that are changed by \pm 2 -fold with an adjusted $P$ value of $<0.05$. (B and $\mathrm{C}$ ) The expression of hypoxia-related (B) and cell wall-related (C) genes in rox $1 \Delta$ mutant relative to WT under conditions indicated above. GPI, glycophosphatidylinositol.

the upc $2 A \Delta$ background are direct targets of Upc2A. This observation further supports the conclusion that Upc2A-independent pathways also regulate Upc2A targets in the presence of fluconazole and that Rox 1 must still play a repressive role in the presence of fluconazole.

Relative to wild type in fluconazole, the $\operatorname{rox} 1 \Delta$ upc $2 A \Delta$ mutant has 157 genes that are upregulated (Fig. $7 C$ and D). Both $u p c 2 A \Delta$ and rox $1 \Delta$ mutants also have significant numbers of genes upregulated in the presence of fluconazole, 159 and 93, respectively. It seems likely that many of the genes that are upregulated in the fluconazoletreated $u p c 2 A \Delta$ strain are related to a compensatory response to the severe sterol stress of those cells. Consistent with that hypothesis, 15 cell wall genes are upregulated in the fluconazole-treated $u p c 2 A \Delta$ cells (corrected $P=0.04$ ). These include the cell wall integrity pathway (CWIP) mitogen-activated protein kinase (MAPK) Slt2, a gene known to be induced and activated by sterol stress (28), along with FKS2, the gene encoding a catalytic subunit of $1,3-\beta$-glucan synthase, which is a gene upregulated by activation of Slt2. Of the 153 genes that are upregulated in the upc $2 A \Delta$ mutant, only $40(26 \%)$ are among the 158 genes that are upregulated in the double mutant in the presence of fluconazole (Fig. 7D). This set is no longer enriched for cell wall genes, and indeed, 
A

Fluconazole-treated rox $1 \triangle$ upc2A $\triangle$ relative to upc2A $\Delta$

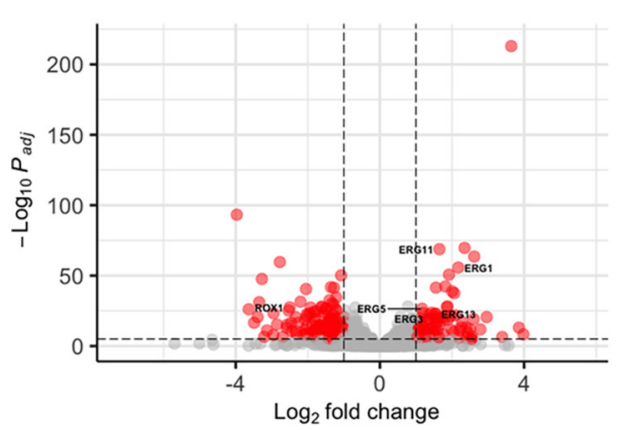

C

Fluconazole-treated rox $1 \Delta$ upc $2 A \Delta$ relative to WT

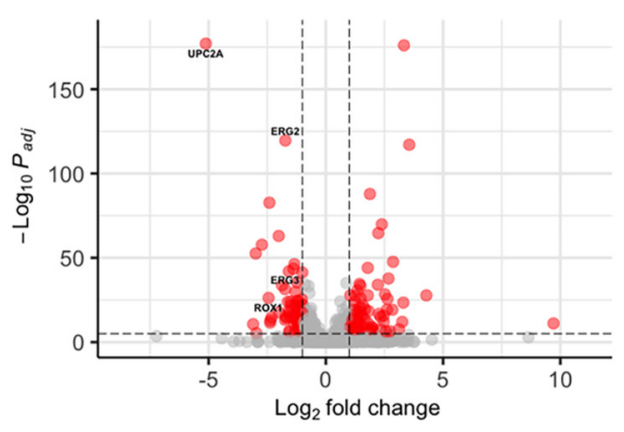

B

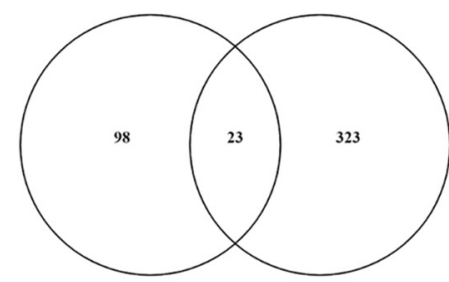

Genes up-regulated Upc2A targets in upc $2 A \triangle \operatorname{rox} 1 \triangle$ relative to upc2A $\triangle$ by ChIP-Seq
D Up-regulated genes relative to fluconazole-treated WT

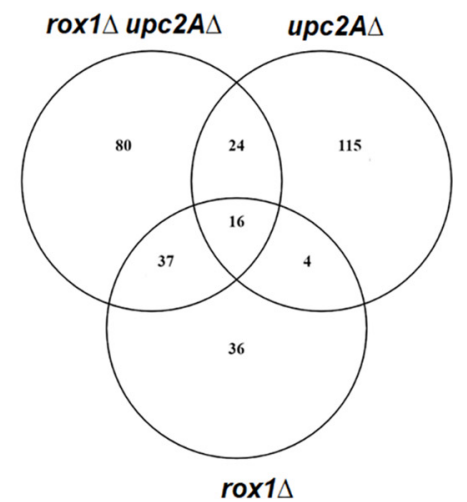

FIG 7 Transcriptional profile of the $\operatorname{rox} 1 \Delta$ upc2A $2 A$ mutant relative to WT and to the single mutant strains in the presence of fluconazole. The strains were incubated in YPD $+8 \mu \mathrm{g} / \mathrm{ml}$ fluconazole for 4 h. (A) Volcano plot comparing $\operatorname{rox} 1 \Delta$ upc $2 A \Delta$ to upc2As strains. Red indicates genes that are changed by \pm 2 -fold with a corrected $P$ value of $<0.05$. ERG genes meeting these criteria are labeled. (B) Venn diagram of genes upregulated in the rox $1 \Delta$ upc2AD mutant relative to $W T$ in the presence of fluconazole compared to Upc2A targets identified by ChIP-seq (27). (C) Comparison of the rox1 $1 \Delta$ upc $2 A \Delta$ mutant to WT in the presence of fluconazole. (D) Venn diagram of genes upregulated in fluconazole-treated $\operatorname{rox} 1 \Delta$, upc2A , and $\operatorname{rox} 1 \Delta$ upc2A $A$ mutants.

none of the 15 cell wall genes remain upregulated. These observations are consistent with the hypothesis that the large set of genes upregulated in the upc $2 A \Delta$ strain represents an exaggerated compensatory response to sterol stress that is then largely eliminated by the deletion of ROX1.

Overall, our transcriptional profiling supports the conclusion that Rox 1 function is reasonably well conserved between S. cerevisiae and C. glabrata. Deletion of Rox1 suppresses the upc2A $A$ fluconazole hypersusceptibility phenotype, at least in part, by derepressing $E R G$ gene expression through a Upc2A-independent pathway. Finally, fluconazole-treated upc $2 A \Delta$ cells display a compensatory transcriptional response that is no longer induced in the fluconazole-treated $\operatorname{rox} 1 \Delta$ upc2A $\Delta$ double mutant.

\section{DISCUSSION}

The ergosterol biosynthesis pathway is not only an essential component of fungal membrane biology but also the target of two of the three classes of drugs that are used to treat human fungal infections. Despite its centrality to fungal biology and antifungal drug therapy, our models for the regulation of ergosterol biosynthesis are relatively limited, particularly in the case of pathogenic fungi (13). In general, current understanding of the transcriptional regulation of ERG gene expression is limited almost entirely to the role of SREB transcription factors or functionally homologous, $\mathrm{Zn}$ 
cluster transcription factors such as Upc2A (14). Although many ERG genes are known to be essential, none of the TFs that regulate their expression are essential, indicating that additional factors are likely to contribute to the regulation of ERG genes. In addition, overexpression experiments in S. cerevisiae have shown that inappropriately high expression of ERG genes reduces fitness, likely due to the fact that sterols can be toxic to the cell (29). As such, it seemed likely that negative regulators of sterol biosynthesis may be required to maintain sterol homeostasis. Our screen was designed to identify either gain-of-function mutants in positive regulators of ERG gene expression or lossof-function mutants in negative regulators and yielded two negative regulators of $E R G$ gene expression: Rox1 and potentially Cst6.

Based on previous work regarding the roles of ScRox 1 and ScUpc2/Ecm 22 in the regulation of hypoxia and sterol stress, the ability of rox $1 \Delta$ to suppress the upc $2 A \Delta$ phenotype is somewhat unexpected. Specifically, in S. cerevisiae, ScRox1 represses many genes that are activated by $S \mathrm{CUpc} 2$, and thus, loss of the Rox 1 repressor should require the presence of the cognate activator for shared genes to be expressed (23). In the presence of fluconazole, the deletion of $R O X 1$ in the upc $2 A \Delta$ strain leads to normalization of some, but not all, ERG gene expression and increased overall levels of ergosterol relative to WT C. glabrata cells. Accordingly, there must be a transcription factor in addition to Upc2A that is able to activate the expression of at least some ERG genes in the presence of fluconazole; otherwise, loss of the repressor Rox 1 would not be able to restore growth during sterol stress because UPC2A was deleted in the parental strains used in the screen. This factor also must be sufficient to restore growth under hypoxic conditions to upc $2 A \Delta$ mutants because the rox $1 \Delta$ mutation suppresses the hypersensitivity of the upc $2 A \Delta$ mutant to those conditions.

Upc2A is currently the only factor that has been directly implicated in the regulation of ERG genes in C. glabrata (9-14). Although Upc2B would seem a logical candidate for this factor, it has not been shown to play a role in the regulation of ERG genes (10). In the fluconazole-treated $u p c 2 A \Delta$ rox $1 \Delta$ mutants, UPC2B expression is modestly reduced (see Table S2 in the supplemental material). This is consistent with previously published data indicating that Upc2A is required for induction of UPC2B expression (10). We cannot completely rule out the possibility that Upc $2 B$ contributes to the regulation of ERG gene expression in the absence of UPC2A, but the current data seem more consistent with an alternative explanation.

A second candidate factor is Hap1 which, based on the large-scale genetic phenotyping project, is required for WT growth in fluconazole (17). HAP1 is upregulated in the rox $1 \Delta$ mutant in the absence of fluconazole and is expressed near WT levels in the presence of fluconazole in the upc $2 A \Delta \operatorname{rox} 1 \Delta$ double mutant (Table S2). Hap1 is also involved in the expression of ERG genes under unstressed conditions in S. cerevisiae (30). Certainly, other factors with less obvious effects on fluconazole stress could also be involved.

Another question raised by our observations is, why is the putative missing ERG gene regulating factor not activated in the presence of fluconazole to compensate for the upc $2 A \Delta$ mutation? The genetic interaction of upc $2 A \Delta$ and $\operatorname{rox} 1 \Delta$ implies that the presence of Rox 1 prevents this factor from positively regulating $E R G$ gene expression in the absence of Upc2A. The data also suggest that Rox 1 may still repress the expression of some genes during treatment with fluconazole or under low-oxygen conditions. However, the only transcription factor that is upregulated in the $\operatorname{rox} 1 \Delta$ upc $2 A \Delta$ mutant is Stb5, which has been shown as a negative regulator of azole susceptibility by decreasing expression of CDR1 and other efflux pumps (31). Another possibility is that Upc2A may be needed to fully displace Rox 1 from promoters and Rox1, in turn, may directly interfere with the binding of this other positive activator to ERG gene promoters. Additional work will be needed to identify the missing positive regulator and understand the mechanism for the interactions between the positive and negative regulators of ergosterol biosynthesis.

The biochemical mechanism by which the $\operatorname{rox} 1 \Delta$ mutation suppresses upc $2 A \Delta$ fluconazole hypersusceptibility is by restoring ERG11 and other ERG genes to wild-type or 
nearly wild-type levels in the presence of fluconazole and, thereby, leading to increased ergosterol content in $\operatorname{rox} 1 \Delta$ upc $2 A \Delta$ cells relative to upc $2 A \Delta$ cells. The sterol profile of the fluconazole-treated double mutant differs from WT, indicating that normal flux through the ergosterol pathway has not been reestablished. Specifically, sterols that are dependent on Erg3 and Erg6 are reduced while the sterols that serve as the Erg3 and Erg6 substrates are increased. This correlates with reduced expression of the ERG3 and ERG6 genes in the double mutant. The fact that the double mutant has ERG11 expression restored to WT levels but not ERG3 and ERG6 further emphasizes the complexity of ergosterol biosynthesis gene regulation and that Upc2A and Rox 1 are not simply on/off switches for a common set of genes.

Furthermore, this apparent reduction in Erg3 and Erg6 leads to a reduction in the toxic sterol $14 \alpha$ methyl-ergosta-8,24(28)-dien-3 $\beta, 6 \alpha$-diol, which accumulates in cells treated with the Erg11-inhibiting azoles (25). Thus, the rox $1 \Delta$ upc $2 A \Delta$ strain has both an increase in ergosterol and a decrease in the toxic sterol relative to upc $2 A \Delta$ and wildtype cells. This not only provides a mechanism for the restoration of wild-type azole susceptibility through deletion of $R O X 1$ in the upc $2 A \Delta$ mutant but also indicates that changes in the relative expression of ERG genes can lead to profound changes in azole susceptibility. In principle, these data suggest that single nucleotide polymorphisms (SNPs) in the promoter regions of ERG genes or epigenetic alterations in gene expression could lead to changes in azole susceptibility without any change to protein coding sequences.

Lastly, we consider the implications of our findings in C. glabrata for C. albicans, which, as noted in this section, has a number of distinctions with respect to the genetic regulation of fluconazole susceptibility. Most notably, the Rox1 homolog in C. albicans is Rfg1, and although it is an HMG class repressor, it has no role in the suppression of hypoxia genes. Instead, it represses filamentation $(32,33)$. Although a Upc2A homolog is present and has been shown to function very similarly, there are no Upc2B/ScEcm 33 homologs in C. albicans. Taken together, our data provide strong evidence for the conclusion that, although the ergosterol biosynthesis pathway is highly conserved among pathogenic and model yeasts, significant changes have occurred in terms of its transcriptional regulation. A more detailed understanding of these distinctions may provide additional insights into the possible mechanisms of resistance to inhibitors of ergosterol biosynthesis.

In summary, our genetic suppressor study provides new insights into the regulation of ergosterol biosynthesis and fluconazole response pathways in C. glabrata. These data highlight the complex nature of the transcriptional pathways that maintain ergosterol homeostasis during normal growth and under sterol stress. Future studies will further explore mechanisms by which the putative Rox 1 suppressor pathway is regulated and how Cst6 affects ergosterol biosynthesis in C. glabrata.

\section{MATERIALS AND METHODS}

Media, reagents, and general methods. Yeast-peptone-dextrose (YPD) and synthetic complete medium (SCM) with amino acid dropout/supplements were prepared using standard recipes (34) for both liquid and plate ( $2 \%$ agar)-based media. Strains were streaked from frozen stocks onto YPD plates and incubated at $30^{\circ} \mathrm{C}$. For all experiments, strains were precultured in liquid medium with shaking at $30^{\circ} \mathrm{C}$ overnight. Fluconazole was obtained as a powder from Sigma.

Strain and plasmid construction. The parental upc $2 A \Delta$ strain for the laboratory evolution experiment was constructed in the BG2 background (17) as described in the work of Vu et al. using the recyclable nourseothricin (NAT) cassette flanked by $1 \mathrm{~kb}$ of homology to the upstream and downstream regions of UPC2A (18). Correct integration was confirmed by PCR as was the absence of the UPC2A open reading frame (ORF). The rox1 $1 \Delta$ upc2A $\Delta$ mutant was constructed in a similar manner in the KK2001 background by deleting the ROX1 coding region from the previously reported upc2ADERG11-hemagglutinin (HA) strain (27).

C. glabrata transformation. Transformations of $C$. glabrata were performed using the lithium acetate method (18). After heat shock, cells were grown overnight at $30^{\circ} \mathrm{C}$ at $200 \mathrm{rpm}$ for drug selection. Overnight cultures were spun down and plated on YPD plates supplemented with $100 \mu \mathrm{g} / \mathrm{ml}$ of nourseothricin (NAT) (Jena Bioscience, Jena, Germany). Colonies were purified by plating on YPD plates containing $200 \mu \mathrm{g} / \mathrm{ml}$ NAT.

Experimental evolution of suppressors of upc2A $\Delta$ fluconazole hypersusceptibility. Three independent colonies of the upc2A $\Delta$ mutant in the His-BG2 background were precultured overnight in YPD. The three cultures were used to inoculate three separate 96 -well plates at 1,000 CFU/well in YPD 
containing $0.25 \mu \mathrm{g} / \mathrm{ml}$ fluconazole ( $1 / 4 \mathrm{MIC}$ for this strain). The plates were incubated at $30^{\circ} \mathrm{C}$ until the wells were saturated. The cultures were serially passaged by transferring $2 \mu \mathrm{l}$ of each well to a fresh plate containing $2 \times$ the fluconazole concentration as the previous plate up to a final concentration of $64 \mu \mathrm{g} / \mathrm{ml}$. Surviving lineages were plated on YPD, and random colonies from each were serially streaked on YPD without fluconazole. The ability of the strains to grow with $64 \mu \mathrm{g} / \mathrm{ml}$ fluconazole was retested. From this experiment, 12 independent strains derived from the upc $2 A \Delta$ mutant with the ability to grow with $64 \mu \mathrm{g} / \mathrm{ml}$ were isolated: the table in Fig. 2A shows the names of the resistant strains. The genomes of the strains were sequenced using Illumina at the University of lowa Genomics Core. The genomes were assembled, and SNPs were called using the Lasergene platform.

Spot dilution assay. Strains were grown overnight at $30^{\circ} \mathrm{C}$ and $200 \mathrm{rpm}$ in liquid YPD, SCM, or SCM without methionine and cysteine. For YPD and YPD + fluconazole, the strains were diluted to an optical density at $600 \mathrm{~nm}\left(\mathrm{OD}_{600}\right)$ of 1 and plated with 10 -fold serial dilutions. Plates were incubated at $30^{\circ} \mathrm{C}$ or $37^{\circ} \mathrm{C}$ for 1 to 3 days before photographing them.

Hypoxia growth assay. Cells were grown in liquid YPD overnight at $30^{\circ} \mathrm{C}$ and $200 \mathrm{rpm}$ and diluted to an OD of 1. Cells were plated on YPD and serially diluted 10-fold for spot dilutions. Plates were sealed in an BD GasPak EZ anaerobe gas generating pouch system with indicator and placed in a $30^{\circ} \mathrm{C}$ incubator or placed directly in an incubator. Plates were captured after $48 \mathrm{~h}$.

Western blot analysis. Strains were incubated in YPD or with YPD in fluconazole for $4 \mathrm{~h}$. Proteins were extracted as previously described (27), resuspended in $120 \mu$ l of urea-SDS sample buffer (8 M urea, $5 \% \mathrm{SDS}, 1 \%$ 2-mercaptoethanol, $40 \mathrm{mM}$ Tris- $\mathrm{HCl}, \mathrm{pH} 8.0$, bromophenol blue), and incubated at $37^{\circ} \mathrm{C}$ for $3 \mathrm{~h}$ by occasional vortexing. Aliquots of $12 \mu \mathrm{l}$ were resolved on a precast ExpressPlus 4 to $12 \%$ gradient gel (GenScript catalog no. M41212). Proteins were electroblotted to an nitrocellulose membrane, blocked with $5 \%$ nonfat dry milk. Membrane was probed with polyclonal anti-Cdr1 antibody (27) in the final dilution of 1:1,000 overnight at $4^{\circ} \mathrm{C}$ and with $12 \mathrm{G} 10$ anti-alpha-tubulin monoclonal antibody (Developmental Studies Hybridoma Bank at the University of lowa) in the final dilution of 1:5,000 for 15 $\mathrm{min}$ at room temperature. Secondary Li-Cor antibodies IRD dye 680RD goat anti-rabbit (catalog no. 92668021) and IRD dye 800LT goat anti-mouse (catalog no. 926-32210) were used in the final dilution of 1:10,000. A Li-Cor infrared imaging system (Li-Cor; application software version 3.0) was used to detect the signal. The data represent results of two biologically independent experiments.

Sterol profiling. Overnight cultures from single colonies of $C$. glabrata strains were used to inoculate $20 \mathrm{ml} \mathrm{YPD} \mathrm{(starting} \mathrm{OD}_{600}$ of 0.20 ) in the absence (dimethyl sulfoxide [DMSO] control, $1 \%$ [vol/vol]) or presence of $8 \mu \mathrm{g} / \mathrm{ml}$ fluconazole (stock prepared in DMSO; final concentration, 1\% [vol/vol] DMSO). Cultures were grown at $30^{\circ} \mathrm{C}$ for $16 \mathrm{~h}$ at $180 \mathrm{rpm}$. Cells were then pelleted and washed with double-distilled water $\left(\mathrm{ddH}_{2} \mathrm{O}\right)$ before splitting each sample for sterol extraction and dry weight determination. Sterols were extracted and derivatized as previously described (35). An internal standard of $5 \mu \mathrm{g}$ of cholesterol was added to each sample, and lipids were saponified using alcoholic $\mathrm{KOH}$ and nonsaponifiable lipids extracted with hexane. Samples were dried in a vacuum centrifuge and were derivatized by the addition of $0.1 \mathrm{ml} \mathrm{N,O-bis(trimethylsilyl)trifluoroacetamide/trimethylchlorosilane} \mathrm{(99:1;} \mathrm{Sigma)} \mathrm{and}$ $0.3 \mathrm{ml}$ anhydrous pyridine (Sigma) and heating at $80^{\circ} \mathrm{C}$ for $2 \mathrm{~h}$. Trimethylsilyl-derivatized sterols were analyzed and identified using gas chromatography-mass spectrometry (GC-MS) (Thermo 1300 GC coupled to a Thermo ISQ mass spectrometer; Thermo Scientific) and Xcalibur software (Thermo Scientific). The retention times and fragmentation spectra for known standards were used to identify sterols. Integrated peak areas were determined to calculate the percentage of total sterols. Ergosterol quantities were determined using standard curves of peak areas of known quantities of cholesterol and ergosterol. Sterol composition and ergosterol quantities were calculated as the mean from three replicates. The statistical significance of the differences between strains was determined using the means and standard errors of the means and Student's $t$ test with $P<0.05$ indicating statistical significance. The complete sterol data and summary are provided in Table $\mathrm{S} 1$ in the supplemental material.

Transcriptional analysis by qPCR. Strains were incubated overnight in liquid YPD at $30^{\circ} \mathrm{C}$ at $200 \mathrm{rpm}$, back-diluted into fresh YPD, and grown to mid-log phase. Cells were harvested at mid-log phase and resuspended into fresh YPD or YPD with $8 \mu \mathrm{g} / \mathrm{ml}$ of fluconazole. Cultures were incubated for $4 \mathrm{~h}$ and harvested, and total RNA was isolated with the MasterPure yeast RNA purification kit. The RNA was reverse transcribed using the iscript CDNA synthesis kit (170-8891; Bio-Rad), and quantitative reverse transcription-PCR (qPCR) was performed using IQ SyberGreen supermix (170-8882; Bio-Rad). ACT1 expression was used as the normalization standard, and relative expression between strains and conditions was determined using the cycle threshold $\left(\Delta \Delta C_{T}\right)$ method. Experiments were performed in biological duplicate with technical triplicates, and the statistical significance was determined using Student's $t$ test with a significance limit of $P<0.05$.

RNA sequencing methods. The strains were grown with and without fluconazole as described for single-gene expression analysis by qRT-PCR. RNA sequencing was performed using Illumina MiSeq for stranded mRNA. Libraries were prepared with paired-end adapters using Illumina chemistries per manufacturer's instructions, and sequencing of libraries was performed with read lengths of approximately $150 \mathrm{bp}$ with at least 50 million reads per sample. RNA sequencing reads were imported into CLC Genomics Workbench 20.0. Sequences were trimmed and aligned to reference sequence (https://www .ncbi.nlm.nih.gov/assembly/GCF_000002545.3) ASM254v2_genomic-1 with paired reads counted as one. Mismatch, insertion, and deletion costs were set to CLC default parameters. Differential expression analysis for whole-transcriptome RNA-Seq was performed using CLC Genomics Workbench. The Wald test was used in all group pairs against the indicated control group. Statistics were based on the fit of a generalized linear model with a negative binomial distribution. C. glabrata gene annotations were obtained from the Candida Genome Database. The fold changes and corrected $P$ values for all genes and all 
conditions are provided in Table S2. GO term analysis was performed using the FungiDB website using

Benjamini-Hochberg-corrected $P$ values of $<0.05$ to identify significantly enriched gene sets.

Data availability. Study data have been deposited in the NCBI Gene Expression Omnibus under accession number GSE191145.

\section{SUPPLEMENTAL MATERIAL}

Supplemental material is available online only.

FIG S1, TIF file, $0.2 \mathrm{MB}$.

TABLE S1, XLSX file, $0.1 \mathrm{MB}$.

TABLE S2, XLSX file, 1.9 MB.

\section{ACKNOWLEDGMENTS}

This work was supported by NIH grants 5R01Al52494 (W.S.M.-R.) and 7R01Al131620 (P.D.R.). The funders had no role in study design, data collection and interpretation, or the decision to submit the work for publication.

\section{REFERENCES}

1. McCarty TP, Pappas PG. 2016. Invasive candidiasis. Infect Dis Clin North Am 30:103-124. https://doi.org/10.1016/j.idc.2015.10.013.

2. Pfaller MA, Andes DR, Diekema DJ, Horn DL, Reboli AC, Rotstein C, Franks B, Azie NE. 2014. Epidemiology and outcomes of invasive candidiasis due to non-albicans species of Candida in 2,496 patients: data from the Prospective Antifungal Therapy (PATH) registry 2004-2008. PLoS One 9: e101510. https://doi.org/10.1371/journal.pone.0101510.

3. Lyman M, Forsberg K, Reuben J, Dang T, Free R, Seagle EE, Sexton DJ, Soda $E$, Jones H, Hawkins D, Anderson A, Bassett J, Lockhart SR, Merengwa $E$, lyengar $P$, Jackson BR, Chiller T. 2021. Notes from the field: transmission of pan-resistant and echinocandin-resistant Candida auris in healthcare facilities-Texas and the District of Columbia. MMWR Morb Mortal Wkly Rep 70:1022-1023. https://doi.org/10.15585/mmwr.mm7029a2.

4. Berman J, Krysan DJ. 2020. Drug resistance and tolerance in fungi. Nat Rev Microbiol 18:319-331. https://doi.org/10.1038/s41579-019-0322-2.

5. Alexander BD, Johnson MD, Pfeiffer CD, Jimenez-Ortigosa C, Catania J, Booker R, Castanheira M, Messer SA, Perlin DS, Pfaller MA. 2013. Increasing echinocandin resistance in Candida glabrata: clinical failure correlates with presence of FKS mutations and elevated minimum inhibitory concentrations. Clin Infect Dis 56:1724-1732. https://doi.org/10.1093/cid/cit136.

6. Nishimoto AT, Sharma C, Rogers PD. 2020. Molecular and genetic basis of azole antifungal resistance in the opportunistic fungus Candida albicans. J Antimicrob Chemother 75:257-270. https://doi.org/10.1093/jac/dkz400.

7. Pristov KE, Ghannoum MA. 2019. Resistance of Candida to azoles and echinocandins worldwide. Clin Microbiol Infect 25:792-798. https://doi .org/10.1016/j.cmi.2019.03.028.

8. Simonicova L, Moye-Rowley WS. 2020. Functional information from clinically-derived forms of the Candida glabrata Pdr1 transcription factor. PLoS Genet 16:e1009005. https://doi.org/10.1371/journal.pgen.1009005.

9. Whaley SG, Caudle KE, Vermitsky JP, Chadwick SG, Toner G, Barker KS, Gygax SE, Rogers PD. 2014. UPC2A is required for high-level antifungal resistance in Candida glabrata. Antimicrob Agents Chemother 58:4543-4554. https://doi.org/10.1128/AAC.02217-13.

10. Nagi M, Nakayama H, Tanabe K, Bard M, Aoyama T, Okano M, Higashi S, Ueno K, Chibana H, Niimi M, Yamagoe S, Umeyama T, Kajiwara S, Ohno H, Miyazaki Y. 2011. Transcription factors CgUPC2A and CgUPC2B regulate ergosterol biosynthetic genes in Candida glabrata. Genes Cells 16:80-89. https://doi.org/10.1111/j.1365-2443.2010.01470.x.

11. Li QQ, Tsai HF, Mandal A, Walker BA, Noble JA, Fukuda Y, Bennett JE. 2018. Sterol uptake and sterol biosynthesis act coordinately to mediate antifungal resistance in Candida glabrata under azole and hypoxic stress. Mol Med Rep 17:6585-6597. https://doi.org/10.3892/mmr.2018.8716.

12. Vik A, Rine J. 2001. Upc2p and Ecm22p, dual regulators of sterol biosynthesis in Saccharomyces cerevisiae. Mol Cell Biol 21:6395-6405. https://doi .org/10.1128/MCB.21.19.6395-6405.2001.

13. Jordá T, Puig S. 2020. Regulation of ergosterol biosynthesis in Saccharomyces cerevisiae. Genes (Basel) 11:795. https://doi.org/10.3390/genes11070795.

14. Maguire SL, Wang C, Holland LM, Brunel F, Neuvéglise C, Nicaud JM, Zavrel M, White TC, Wolfe KH, Butler G. 2014. Zinc finger transcription factors displaced SREBP proteins as the major sterol regulators during
Saccharomycotina evolution. PLoS Genet 10:e1004076. https://doi.org/10 .1371/journal.pgen.1004076.

15. Deckert J, Khalaf RA, Hwang SM, Zitomer RS. 1999. Characterization of the DNA binding and bending HMG domain of the yeast hypoxic repressor Rox1. Nucleic Acids Res 27:3518-3526. https://doi.org/10.1093/nar/27.17 .3518 .

16. Liu G, Bergenholm D, Nielsen J. 2016. Genome-wide mapping of binding sites reveals multiple biological functions of the transcription factor Cst6p in Saccharomyces cerevisiae. mBio 7:e00559-16. https://doi.org/10 .1128/mBio.00559-16.

17. Schwarzmüller T, Ma B, Hiller E, Istel F, Tscherner M, Brunke $S$, Ames $L$, Firon A, Green B, Cabral V, Marcet-Houben M, Jacobsen ID, Quintin J, Seider K, Frohner I, Glaser W, Jungwirth H, Bachellier-Bassi S, Chauvel M, Zeidler U, Ferrandon D, Gabaldón T, Hube B, d'Enfert C, Rupp S, Cormack B, Haynes K, Kuchler K. 2014. Systematic phenotyping of a large-scale Candida glabrata deletion collection reveals novel antifungal tolerance genes. PLoS Pathog 10:e1004211. https://doi.org/10.1371/journal.ppat.1004211.

18. Vu BG, Moye-Rowley WS. 2018. Construction and use of a recyclable marker to examine the role of major facilitator superfamily protein members in Candida glabrata drug resistance phenotypes. mSphere 3:e0009918. https://doi.org/10.1128/mSphere.00099-18.

19. Paul S, Bair TB, Moye-Rowley WS. 2014. Identification of genomic binding sites for Candida glabrata Pdr1 transcription factor in wild-type and rho0 cells. Antimicrob Agents Chemother 58:6904-6912. https://doi.org/10 .1128/AAC.03921-14.

20. d'Enfert C, Janbon G. 2016. Biofilm formation in Candida glabrata: what have we learnt from functional genomics approaches? FEMS Yeast Res 16:fov111. https://doi.org/10.1093/femsyr/fov111.

21. Pohlers S, Martin R, Krüger T, Hellwig D, Hänel F, Kniemeyer O, Saluz HP, Van Dijck P, Ernst JF, Brakhage A, Mühlschlegel FA, Kurzai O. 2017. Lipid signaling via $\mathrm{Pkh} 1 / 2$ regulates fungal $\mathrm{CO}_{2}$ sensing through the kinase Sch9. mBio 8:e02211-16. https://doi.org/10.1128/mBio.02211-16.

22. Vandeputte P, Pradervand S, Ischer F, Coste AT, Ferrari S, Harshman K, Sanglard D. 2012. Identification and functional characterization of Rca1, a transcription factor involved in antifungal susceptibility and host response in Candida albicans. Eukaryot Cell 11:916-931. https://doi.org/10.1128/EC .00134-12.

23. Kwast KE, Lai LC, Menda N, James DT, III, Aref S, Burke PV. 2002. Genomic analysis of anaerobically induced genes in Saccharomyces cerevisiae: functional roles of Rox 1 and other factors in mediating the anoxic response. J Bacteriol 184:250-265. https://doi.org/10.1128/JB.184.1.250-265.2002.

24. Henry KW, Nickels JT, Edlind TD. 2002. ROX1 and ERG regulation in Saccharomyces cerevisiae: implications for antifungal susceptibility. Eukaryot Cell 1:1041-1044. https://doi.org/10.1128/EC.1.6.1041-1044.2002.

25. Kelly SL, Lamb DC, Corran AJ, Baldwin BC, Kelly DE. 1995. Mode of action and resistance to azole antifungals associated with the formation of 14 alpha-methylergosta-8,24(28)-dien-3 beta, 6 alpha-diol. Biochem Biophys Res Commun 207:910-915. https://doi.org/10.1006/bbrc.1995.1272.

26. Inukai T, Nagi M, Morita A, Tanabe K, Aoyama T, Miyazaki Y, Bard M, Nakayama H. 2015. The mannoprotein TIR3 (CAGL03872g) is required for 
sterol uptake in Candida glabrata. Biochim Biophys Acta 1851:141-151. https://doi.org/10.1016/j.bbalip.2014.11.002.

27. Vu BG, Stamnes MA, Li Y, Rogers PD, Moye-Rowley WS. 2021. Azole resistance is mediated by integration of sterol gene regulation and membrane transporter production by the zinc cluster-containing transcription factor Upc2A in Candida glabrata. PLoS Genet 17:e1009582. https://doi.org/10 .1371/journal.pgen.1009582.

28. Borah S, Shivarathri R, Kaur R. 2011. The Rho1 GTPase-activating protein $\mathrm{CgBem} 2$ is required for survival of azole stress in Candida glabrata. J Biol Chem 286:34311-34324. https://doi.org/10.1074/jbc.M111.264671.

29. Bhattacharya S, Esquivel BD, White TC. 2018. Overexpression or deletion of ergosterol biosynthesis genes alters the doubling time, response to stress agents, and drug susceptibility in Saccharomyces cerevisiae. mBio 9: e01291-18. https://doi.org/10.1128/mBio.01291-18.

30. Tamura K, Gu Y, Wang Q, Yamada T, Ito K, Shimoi H. 2004. A hap1 mutation in a laboratory strain of Saccharomyces cerevisiae results in decreased expression of ergosterol-related genes and cellular ergosterol content compared to sake yeast. J Biosci Bioeng 98:159-166. https://doi.org/10.1016/ S1389-1723(04)00260-9.
31. Noble JA, Tsai HF, Suffis SD, Su Q, Myers TG, Bennett JE. 2013. STB5 is a negative regulator of azole resistance in Candida glabrata. Antimicrob Agents Chemother 57:959-967. https://doi.org/10.1128/AAC .01278-12.

32. Kadosh D, Johnson AD. 2001. Rfg1, a protein related to the Saccharomyces cerevisiae hypoxic regulator Rox1, controls filamentation and virulence in Candida albicans. Mol Cell Biol 21:2496-2505. https://doi.org/10 .1128/MCB.21.7.2496-2505.2001.

33. Khalaf RA, Zitomer RS. 2001. The DNA binding protein Rfg1 is a repressor of filamentation in Candida albicans. Genetics 157:1503-1512. https://doi .org/10.1093/genetics/157.4.1503.

34. Homann OR, Dea J, Noble SM, Johnson AD. 2009. A phenotypic profile of the Candida albicans regulatory network. PLoS Genet 5:e1000783. https:// doi.org/10.1371/journal.pgen.1000783.

35. Parker JE, Warrilow AG, Cools HJ, Fraaije BA, Lucas JA, Rigdova K, Griffiths WJ, Kelly DE, Kelly SL. 2013. Prothioconazole and prothioconazole-desthio activities against Candida albicans sterol 14- $\alpha$-demethylase. Appl Environ Microbiol 79:1639-1645. https://doi.org/10.1128/ AEM.03246-12. 